\title{
Efectos neuroprotectores del bilingüismo contra la demencia Un estudio de morfometría basada en el voxel
}

\author{
Lidón Marín Marín \\ lidon.marin@uji.es \\ Víctor Costumero Ramos \\ vcostume@uji.es \\ Naiara Aguirre Vidal \\ naguirre@uji.es \\ César Ávila Rivera \\ avila@psb.uji.es
}

\section{Resumen}

Introducción: Recientes estudios sugieren que el bilingüismo es uno de los factores que contribuye a la reserva cognitiva, ya que las personas bilingües suelen manifestar los síntomas de la enfermedad de Alzheimer (EA) significativamente más tarde que los monolingües. Otros estudios han revelado que los bilingües muestran mayor atrofia e hipometabolismo comparados con los monolingües, pese a tener el mismo diagnóstico. Sin embargo, todas estas investigaciones incluyen participantes bilingües y monolingües que muestran diferencias en nivel educativo, nivel socioeconómico o estatus de inmigración. El objetivo de este trabajo es investigar las bases neurales del supuesto efecto protector del bilingüismo.

Metodología: Una muestra de 68 pacientes con deterioro cognitivo leve (DCL) fue incluida en el estudio, con 26 bilingües y 42 monolingües. Se recogieron imágenes $3 \mathrm{D}$ de resonancia magnética a partir de las cuales se compararon los volúmenes de sustancia gris de ambos grupos.

Resultados: Bilingües y monolingües no mostraron diferencias significativas en variables demográficas ni en rendimiento cognitivo en pruebas psicológicas. Sin embargo, los bilingües mostraron menor volumen de sustancia gris (mayor atrofia cerebral) que los monolingües en las siguientes áreas: precuneus izquierdo, área de Wernicke izquierda, ínsula/giro de Heschl bilateral y vermis cerebelar bilateral.

Conclusión: Los bilingües, con la misma edad, nivel educativo y manifestación de síntomas de la enfermedad, muestran mayor atrofia de sustancia gris que los monolingües en áreas relevantes para la EA. Esto sugiere que el bilingüismo actúa como un factor protector de la demencia, ya que requiere mayor atrofia cerebral para llegar al mismo estado patológico. 
Palabras clave: bilingüismo, demencia, reserva cognitiva, deterioro cognitivo leve, morfometría basada en el voxel.

\section{Abstract}

Introduction: Evidence from previous studies suggests that bilingualism is one of the factors which contributes to cognitive reserve, by showing that bilingual individuals manifest symptoms of Alzheimer's disease (AD) significantly later than monolinguals. Other evidences revealed that bilinguals show greater amounts of atrophy or hypometabolism compared to monolinguals, despite having the same diagnosis of $A D$ and similar symptoms. However, these previous studies included samples in which bilinguals and monolinguals showed differences in education, immigration and/or socioeconomic status. The aim of this work was to investigate the neural bases of these putative protective effects of bilingualism.

Methods: A sample of 68 patients with mild cognitive impairment $(\mathrm{MCl}), 26$ bilinguals and 42 monolinguals, was included in the study. Whole-brain 3-D magnetic resonance images were collected in order to compare the gray matter volume of both groups.

Results: Bilinguals and monolinguals showed no significant differences in sociodemographic variables nor cognitive performance in neuropsychological testing. However, bilinguals showed less gray matter volume (more brain atrophy) than monolinguals in the following areas: left precuneus, left Wernicke's area, bilateral insula/Heschl's gyrus and bilateral cerebellar vermis.

Conclusion: Bilinguals with the same age, level of education and manifestation of symptoms as monolinguals, show higher levels of gray matter atrophy than monolinguals in relevant areas for AD. This suggests that bilingualism acts as a protective factor against dementia, since it requires more brain atrophy in order to get to the same pathological state.

Keywords: bilingualism, dementia, cognitive reserve, mild cognitive impairment, voxel-based morphometry.

\section{Introducción}

La reserva cognitiva $(R C)$ se refiere a los cambios cerebrales producidos por la práctica de actividad mental compleja, la cual puede mantener la función cognitiva durante el envejecimiento e incluso retrasar la aparición de demencia (Steffener y Stern 2012; Perani et al. 2017). El bilingüismo es uno de los factores que contribuye a la RC (Bialystok, Craik y Luk 2012; Freedman et al. 2014; Guzmán-Velez y Tranel 2015). Se trata de una experiencia lingüística y verbal pero, de la misma manera que otros factores que contribuyen a la RC, también se extiende a actividades no lingüísticas. De esta manera, estudios en países como Canadá (Schweizer, Ware, Fischer, Craik y Bialystok 2012), la India (Alladi et al. 2013) o Italia (Perani et al. 2017) han demostrado que las personas bilingües muestran los primeros síntomas de la enfermedad de alzheimer (EA) de cuatro a cinco años más tarde que los monolingües. Esta demora en la aparición de síntomas supone la base para concluir que el bilingüismo ejerce este factor protector contra la demencia.

Al tratar de investigar los posibles sustratos neurales de este retraso en la aparición de la demencia, pocos estudios previos llevados a cabo demuestran que, ante el mismo nivel de gravedad de la enfermedad y mostrando la misma sintomatología, los pacientes bilingües tienden a mostrar mayor atrofia cerebral que los monolingües 
(Schweizer, Ware, Fischer, Craik y Bialystok 2012; Perani et al. 2017; Duncan et al. 2018). Estos resultados concuerdan con la hipótesis de que el bilingüismo protege contra el deterioro de la demencia y retrasa la aparición de la enfermedad, ya que nos muestran que los bilingües necesitarán mayores cantidades de neuropatología o atrofia, comparados con los monolingües, para que la enfermedad se manifieste. En concreto, en la investigación realizada en Canadá con participantes diagnosticados con EA utilizando imágenes de tomografía axial computarizada (TAC) (Schweizer, Ware, Fischer, Craik y Bialystok 2012), se encontró que el grupo de bilingües mostraba un mayor grado de atrofia que el grupo de monolingües en regiones asociadas con la patología de la enfermedad, pese a mostrar el mismo nivel de funcionamiento cognitivo. En el estudio realizado en Italia mediante el uso de tomografía por emisión de positrones (PET) (Perani et al. 2017), también se encontraron evidencias que concuerdan con esta hipótesis: su grupo de bilingües con EA mostró un nivel mayor de hipometabolismo que el de monolingües. No obstante, no todos los datos obtenidos son consistentes. Recientemente, Duncan et al. (2018) confirmaron una mayor atrofia en zonas temporales mediales en pacientes bilingües con EA, pero el efecto se revertía en pacientes con deterioro cognitivo leve (DCL) monolingües. Este resultado en DCL cuestionaría la hipótesis de reserva cognitiva.

En cuanto al tipo de bilingüismo, todas las investigaciones mencionadas anteriormente presentan una limitación importante: los sujetos monolingües de la muestra presentan diferencias significativas respecto a los bilingües en nivel socioeconómico, años de escolaridad o circunstancias de adquisición de la segunda lengua. Por ejemplo, la mayoría de las poblaciones bilingües consideradas en estos estudios están formadas por personas inmigrantes, que suelen tener una experiencia muy particular con el lenguaje (Bialystok, Craik y Freedman 2007; Alladi et al. 2013). Como Woumans et al. (2015) explican, este tipo de subgrupo no puede ser considerado una muestra al azar o aleatoria, porque presenta características diferentes de la población general y cualquiera de esas diferencias podría haber causado el efecto protector atribuido al bilingüismo. Uno de los estudios previos (Duncan et al. 2018) trató de resolver este problema utilizando una submuestra de participantes no inmigrantes, pero la muestra principal del estudio seguía estando formada por bilingües y monolingües que tenían circunstancias vitales significativamente diferentes. Por todo lo anterior, resulta de vital importancia llevar a cabo estudios en los que bilingües y monolingües sean similares en todas sus características, asegurando de esta manera que ningún otro factor aparte del bilingüismo es el responsable de las diferencias que se encuentran entre los grupos. Sin embargo, la mayoría de los estudios llevados a cabo hasta la fecha utilizan muestras de bilingües y monolingües que no comparten los mismos contextos o tienen un nivel socioeconómico y cultural significativamente diferente. Por ello, nuestro estudio pretende utilizar una muestra en la que el bilingüismo valenciano-castellano no esté asociado a experiencias particulares como la inmigración y en la que pertenecer al grupo de bilingües o monolingües no esté asociado a presentar un nivel socioeconómico más alto o bajo.

El objetivo del presente estudio es investigar el efecto del bilingüismo sobre la manifestación clínica de la demencia y la estructura cerebral en pacientes con DCL, observando las diferencias en volumen de sustancia gris entre bilingües y monolingües, en una muestra obtenida de la misma zona poblacional y controlando la ausencia de diferencias en nivel educativo. Se hipotetiza que se encontrará un mayor nivel de atrofia de sustancia gris en bilingües que en monolingües, pese a mostrar la misma sintomatología de la enfermedad y ser comparables en edad, nivel de estudios y nivel socioeconómico. 


\section{Método}

\section{Participantes}

Sesenta y ocho pacientes con DCL participaron en el estudio (32 mujeres; media de edad $=73,44 \pm 5,81$ ). Todos los individuos fueron reclutados del Hospital General Universitario de Valencia, siguiendo los siguientes criterios de inclusión: 1) quejas de memoria (autoinformadas o confirmadas por un informante); 2) deterioro de memoria objetivo, valorado con el subtest de memoria aditivo verbal del test de Barcelona (Peña-Casanova 2005); 3) independencia en las actividades de la vida diaria esencialmente inalterada; 4) ninguna evidencia de demencia; y 5) puntuación en el Clinical Dementia Rating (CDR; Hughes, Berg, Danziger, Coben y Martin 1982) de 0,5.

Además, se entrevistó a los participantes en relación con su uso del lenguaje. Se dividió a los sujetos dos grupos dependiendo de su grado de bilingüismo: bilingües activos $(N=26)$ y monolingües $(N=42)$. Dicho grado de bilingüismo se calculó teniendo en cuenta el uso y el dominio de su segunda lengua, el catalán/valenciano. Por lo tanto, los sujetos se consideraron bilingües activos si usaban el español y el catalán con frecuencia y alto dominio y monolingües si eran capaces de entender ambos pero solo producían el español. Los dos grupos se emparejaron por nivel de desempeño cognitivo, edad, nivel educativo y nivel socioeconómico, de manera que no hubiera ninguna diferencia significativa entre ellos en estas variables.

Todos los participantes fueron informados de la naturaleza del estudio y dieron su consentimiento antes de su participación. Esta investigación fue aprobada por el Comité de Ética de la Universitat Jaume I y todos los procedimientos utilizados se enmarcan dentro del Código Internacional de Ética de la World Medical Association.

\section{Pruebas neuropsicológicas}

Se utilizó un protocolo estandarizado que consistía en una primera página con datos generales, como diagnóstico, código del sujeto, nombre, mano dominante, años de escolaridad, lengua materna, estado civil, etc. Después se incluyeron una serie de test neuropsicológicos: Mini Mental State Examination (MMSE; Folstein, Folstein y McHugh 1975; Lobo, Saz y Marcos 2002), Functional Activity Questionnaire (FAQ; Pfeffer, Kurosaki, Harrah, Chance y Filos 1982), subtest de dígitos (directos e inversos) de la Wechsler memory scale-III (WMS-III; Wechsler 1997), versión corta del Boston Naming Test (Serrano et al. 2001), versión en español de los test de fluencia fonética y semántica del Controlled Oral Word Association Test (COWAT; Benton, Hamsher y Sivan 1983), subtest de memoria auditivo-verbal dentro del test Barcelona (PeñaCasanova 2005), subtest de memoria lógica (I, II) de la Wechsler Memory Scale-III (WMS-III; Wechsler 1997) y subtest de semejanzas de la escala intelectual de Wechsler para adultos (WAIS-III; Wechsler 1997).

\section{Adquisición de imágenes de resonancia}

La adquisición de las imágenes se realizó en un escáner de resonancia magnética 3T (Siemens Magnetom Trio, Erlangen, Alemania). Los participantes fueron colocados en posición supina en la máquina de resonancia magnética y se les inmovilizó la cabeza mediante cojines para reducir los artefactos producidos por el ruido provocado por el movimiento. Para la adquisición de los datos estructurales, se adquirió un volumen $3 \mathrm{D}$ potenciado en T1 usando una secuencia MP-RAGE (176 cortes; TE = 2,98 ms; $\mathrm{TR}=2300 \mathrm{~ms} ;$ matriz = $256 \times 256$; flip angle = 9o; resolución espacial $=1 \times 1 \times 1 \mathrm{~mm}$ ). 


\section{Preprocesado de las imágenes}

El análisis de morfometría basada en el vóxel (VBM) se realizó mediante la herramienta CAT12 (Computational Anatomy Toolbox; C. Gaser, Jena University Hospital, Jena, Alemania; http://dbm.neuro.uni-jena.de/cat/), implementada para el paquete estadístico SPM12 (Statistical Parametric Mapping 12; Wellcome Trust Centre for Neuroimaging, University College, Londres, Reino Unido; http://www.fil.ion.ucl.ac.uk/spm/), ejecutado en el Matlab R2013b (Mathworks, Sherborn, MA, Estados Unidos). En primer lugar, se realizó un preprocesado estándar que incluyó: corrección de uniformidad en intensidad; normalización a espacio MNI mediante la Diffeomorphic Anatomic Registration Through Exponentiated Lie (DARTEL) (Ashburner 2007); segmentación del tejido en sustancia gris (GM), sustancia blanca y líquido cefalorraquídeo (Ashburner y Friston 2005) y modulación usando los determinantes Jacobianos. El índice de calidad proporcionado por el método automático de garantía de calidad incorporado en el CAT12 fue mayor del $70 \%$ (satisfactorio) en todas las imágenes. Además, las imágenes se revisaron visualmente en busca de cualquier posible artefacto después del preprocesado, para finalmente ser suavizadas con un filtro gaussiano (FWHM) de $8 \mathrm{~mm}$.

\section{Análisis estadístico}

Se estudiaron las diferencias grupales en volumen de sustancia gris utilizando el modelo lineal general implementado en el SPM12. Se utilizó una prueba t para dos muestras, con el criterio estadístico en $p<0,001$ (sin corregir) con un tamaño de cluster de $\mathrm{k}>300$ voxels.

\section{Resultados}

Datos descriptivos y pruebas neuropsicológicas

Los resultados de las comparaciones entre grupos para las pruebas neuropsicológicas, años de escolaridad y edad se incluyen en la tabla 1. No se encontraron diferencias significativas entre bilingües y monolingües en edad, años de escolaridad y ejecución en los diferentes test neuropsicológicos.

Tabla 1

Datos descriptivos y resultados de las pruebas neuropsicológicas para bilingües y monolingües.

\begin{tabular}{cccc} 
& $\begin{array}{c}\text { Bilingües } \\
(\mathrm{n}=26)\end{array}$ & $\begin{array}{c}\text { Monolingües } \\
(\mathrm{n}=42)\end{array}$ & $\mathrm{P}$ \\
\hline Edad & $74,54(5,42)$ & $72,76(6,00)$ & 0,310 \\
Años de escolaridad & $8,43(2,93)$ & $8,35(3,16)$ & 0,641 \\
MMSE & $28,15(2,09)$ & $28,21(1,80)$ & 0,236 \\
Functional Activities & $4,77(2,66)$ & $5,29(4,06)$ & 0,319 \\
Questionnaire & & & \\
Dígitos directos & $5,15(0,61)$ & $4,83(0,79)$ & 0,183 \\
Dígitos inversos & $3,62(0,64)$ & $3,50(0,77)$ & 0,417 \\
Boston & $9,42(1,03)$ & $9,07(1,20)$ & 0,922 \\
Fluencia fonética & $9,04(1,34)$ & $8,86(1,30)$ & 0,987 \\
Fluencia semántica & $10,92(1,90)$ & $10,52(1,81)$ & 0,524
\end{tabular}




\begin{tabular}{cccc} 
Memoria auditivo-verbal & $3,88(0,82)$ & $3,62(0,88)$ & 0,279 \\
Memoria lógica a corto plazo & $10,80(2,15)$ & $10,38(1,77)$ & 0,712 \\
Memoria lógica a largo plazo & $7,08(1,32)$ & $6,95(1,50)$ & 0,864 \\
Semejanzas & $12,54(2,40)$ & $11,40(2,48)$ & 0,718 \\
\hline
\end{tabular}

Morfometría basada en el voxel

Los resultados de la prueba $T$ para dos muestras $(p<0,001$, uncorrected, $k>300)$ evidencian que los bilingües, comparados con los monolingües, presentan un volumen reducido de sustancia gris en las siguientes áreas cerebrales: occipital medio izquierdo (precuneus), occipital superior izquierdo, temporal medio izquierdo (área de Wernicke), temporal superior dere cho (ínsula/Heschl), vermis cerebelar bilateral e ínsula/giro de Heschl izquierdo (véanse la tabla 2 y la figura 1).

Tabla 2

Reducción de volumen cerebral en el grupo de bilingües activos comparados con los monolingües (monolingües > bilingües)

\begin{tabular}{|c|c|c|c|c|}
\hline Regiones & $\begin{array}{l}\text { Coordenadas } \\
\mathrm{MNI}(\mathrm{x}, \mathrm{y}, \mathrm{z})\end{array}$ & $\mathrm{T}$ & K & $\begin{array}{l}\text { Áreas de } \\
\text { Broadmann }\end{array}$ \\
\hline $\begin{array}{l}\text { Occipital medio izquierdo } \\
\text { (precuneus) }\end{array}$ & $-30-8030$ & 4,95 & 1013 & 19,7 \\
\hline $\begin{array}{l}\text { Occipital superior } \\
\text { izquierdo }\end{array}$ & $-21-68 \quad 36$ & 3,72 & & \\
\hline Ínsula/Heschl izquierdo & $-45-115$ & 4,78 & 447 & 13 \\
\hline $\begin{array}{l}\text { Temporal medio izquierdo } \\
\text { (Wernicke) }\end{array}$ & $-65-443$ & 4,47 & 379 & 22,21 \\
\hline $\begin{array}{l}\text { Temporal superior derecho } \\
\text { (ínsula/Heschl) }\end{array}$ & $\begin{array}{lll}48 & -9 & 2\end{array}$ & 4,09 & 336 & 22 \\
\hline $\begin{array}{l}\text { Polo temporal superior } \\
\text { derecho }\end{array}$ & $\begin{array}{lll}62 & 6 & 2\end{array}$ & 3,69 & & \\
\hline Vermis cerebelar 9 & $8-57-36$ & 3,55 & 339 & \\
\hline Cerebelo derecho 9 & $3-47-39$ & 3,50 & & \\
\hline Cerebelo izquierdo 9 & $-3-47-44$ & 3,47 & & \\
\hline
\end{tabular}




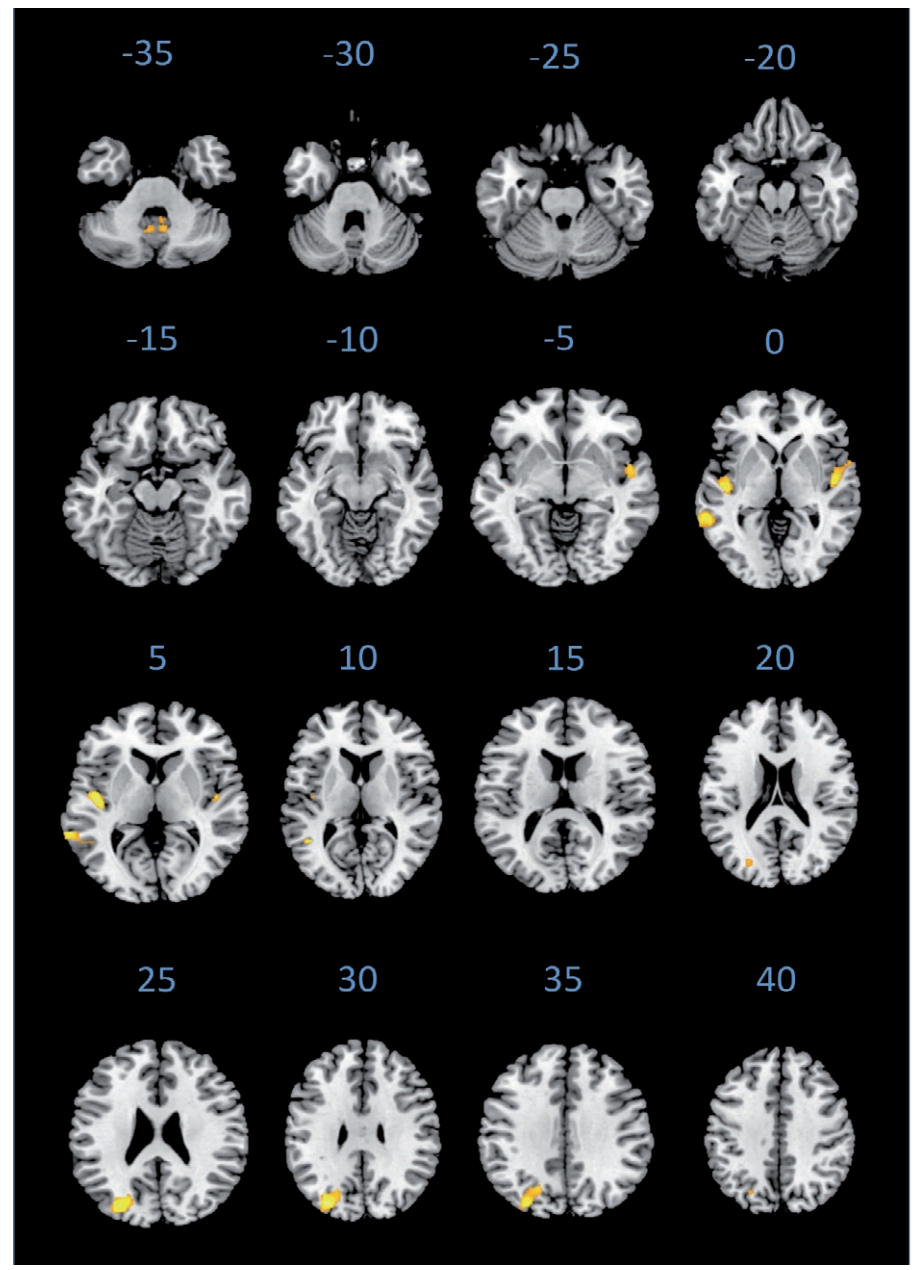

Figura 1. Reducción de volmen de sustancia gris en áreas cerebrales en bilingües comparados con monolingües (monolingües>bilingües)

\section{Discusión y conclusiones}

En el presente estudio investigamos las bases neurales del posible efecto protector del bilingüismo contra la demencia. Para ello, se utilizó una muestra de bilingües y monolingües, comparando sus volúmenes de sustancia gris mediante resonancia magnética funcional. De manera general, los bilingües mostraron menor volumen de sustancia gris que los monolingües en áreas relevantes para la EA, pese a no mostrar diferencias significativas en variables demográficas ni en rendimiento cognitivo en pruebas psicológicas. Por lo tanto, estos resultados sugieren que el bilingüismo podría ejercer un papel protector contra la demencia, debido a que los bilingües necesitan mayor cantidad de atrofia de sustancia gris para mostrar la misma gravedad de la enfermedad a nivel conductual.

En primer lugar, la ausencia de diferencias significativas entre los grupos en las puntuaciones de las pruebas neuropsicológicas administradas sugiere que bilingües y monolingües con DCL se encuentran a un nivel cognitivo comparable. Además, ambos grupos son muy similares en cuanto a edad y años de escolaridad, al contrario que en anteriores estudios, donde los sujetos bilingües eran significativamente mayores que los monolingües (Bialystok, Craik y Freedman 2007; Woumans et al. 2015). Por otra parte, nuestro estudio es el primero en investigar una muestra en la que ambos grupos son comparables en variables socioeconómicas y culturales, con una edad y unas 
circunstancias vitales muy similares. Esto significa que, por ejemplo, al contrario que en anteriores estudios (Bialystok, Craik y Freedman 2007; Alladi et al. 2013; Woumans et al. 2015), nuestro grupo de bilingües no está formado por personas inmigrantes con condiciones vitales diferentes a las de los monolingües, con condiciones de adquisición de la segunda lengua muy especiales o con un nivel de estudios menor que el de los monolingües. En nuestra muestra, bilingües activos y monolingües son similares en muchos aspectos de sus vidas. Esta es una situación muy común en la Comunidad Valenciana y, en concreto, en el territorio donde se realizó nuestro estudio, Valencia, donde bilingües y monolingües comparten los mismos contextos, como colegio, lugar de trabajo o incluso vecindario, con un poder adquisitivo, trasfondo cultural y educación muy similares. Por lo tanto, ambos grupos no presentan diferencias significativas en ningún aspecto que no sea el bilingüismo. En consecuencia, concluimos que las diferencias encontradas en los resultados de morfometría basada en el voxel no se pueden atribuir a otros elementos que no sean la condición de bilingüismo.

Nuestros resultados muestran que el grupo bilingües, con edad, diagnóstico y manifestación de la enfermedad similares a las del grupo monolingüe, muestran una mayor atrofia cerebral. Esto coincide con los resultados de anteriores estudios (Bialystok, Craik y Freedman 2007; Alladi et al. 2013; Woumans et al. 2015; Perani et al. 2017), que sugieren que el bilingüismo podría proteger contra el inicio de la sintomatología de demencia. Por lo tanto, se interpreta que el bilingüismo está actuando como un factor protector, permitiendo a los bilingües mostrar una manifestación conductual de la enfermedad más tarde que los monolingües con el mismo nivel de deterioro cerebral. Si tenemos en cuenta este resultado, junto con la ausencia de diferencias cognitivas y educativas entre grupos, podremos decir que este es el primer estudio publicado que demuestra mayor atrofia cerebral en bilingües en ausencia de otros factores explicativos.

En concreto, nuestros resultados sugieren que las áreas parietooccipitales y temporales izquierdas serían relevantes en la demencia, ya que muestran mayor atrofia de sustancia gris en bilingües que en monolingües con DCL. En primer lugar, encontramos mayor atrofia en el occipital superior y medio izquierdo (precuneus) en bilingües comparados con monolingües, lo cual coincide con estudios anteriores que relacionan atrofia en el precuneus y EA (Karas et al. 2007; Ryu et al. 2010; Perani et al. 2017). El precuneus forma parte de la red neuronal por defecto (RND), la cual se activa durante procesos de alta integración, como la recuperación en memoria (Vannini et al. 2011). Estos resultados plantean la posibilidad de que la atrofia del precuneus pueda estar relacionada con peor ejecución en tareas que requieren recuperación de memoria episódica, lo cual es común en individuos con DCL o EA.

En cuanto a las áreas temporales, nuestros resultados coinciden con los de estudios anteriores (Jack y Petersen 2000; Bidelman, Lowther, Tak y Alain 2017), que muestran que el temporal o el giro de Heschl se encuentran significativamente atrofiados en pacientes con DCL. En concreto, en nuestra investigación vemos cómo los bilingües muestran mayor atrofia que los monolingües en el temporal medio izquierdo (Wernicke) y en el giro de Heschl bilateral. Esto nos sugiere que cambios en áreas auditivas relacionadas con el lenguaje como las anteriormente mencionadas podrían estar relacionadas con la peor ejecución de tareas de lenguaje que se suele observar en pacientes con EA comparados con controles, concordando con resultados que ya fueron descritos años atrás (Martin y Fedio 1983; Murdoch, Chenery, Wilks y Boyle 1987; Illes 1989). Finalmente, estos resultados también nos sugieren que áreas temporales auditivas serían relevantes en $\mathrm{DCL}$ y podrían convertirse en marcadores 0 predictores de la probabilidad de desarrollar EA. 
Por último, nuestros resultados plantean la posibilidad de que el cerebelo pueda tener en papel relevante en la demencia. Sin embargo, no hay muchos estudios previos relacionados con DCL o EA que se centren en el cerebelo y sus diferentes áreas, siendo mencionado solo brevemente y sin darle tanta importancia como a otras áreas. Algunas investigaciones previas mostraron que el cerebelo presenta atrofia tanto en DCL (Thomann et al. 2008) como en EA (Andersen, Andersen y Pakkenberg 2012) y que dicha atrofia se correlaciona con los cambios clínicos durante el desarrollo de la EA (Wegiel et al. 1999). Sin embargo, se necesitaría la realización de futuros estudios para establecer y acordar un rol específico para el cerebelo en la demencia.

A modo de conclusión general, en este estudio se muestra cómo bilingües activos, con la misma edad, años de escolaridad y estadio de demencia que los monolingües, muestran menor volumen de sustancia gris en áreas parietooccipitales y temporales. Esto sugiere que la mayor atrofia en bilingües no puede ser explicada por diferencias entre grupos en edad, nivel educativo o gravedad de la enfermedad, sino solamente por las diferencias en bilingüismo. Por lo tanto, se refuerza la hipótesis de que el bilingüismo podría estar actuando como un factor protector contra la demencia, contribuyendo también a la reserva cognitiva.

\section{Referencias bibliográficas}

Alladi, Suvarna, Thomas H. Bak, Vasanta Duggirala, Bapiraju Surampudi, Makela Shailaja, Anuj K. Shukla, Jaydip Ray Chaudhuri y Subhash Kaul. 2013. «Bilingualism delays age at onset of dementia, independent of education and immigration status». Neurology 81(22): 1938-1944.

Andersen, Kjeld, Brigitte Bo Andersen y Bente Pakkenberg. 2012. "Stereological quantification of the cerebellum in patients with Alzheimer's disease». Neurobiology of aging 33(1): 197.e11-197.e20.

Ashburner, John. 2007. «A fast diffeomorphic image registration algorithm». Neuroimage 38(1): 95-113.

Ashburner, John y Karl J. Friston. 2005. «Unified segmentation». Neuroimage 26(3): 839-851.

Benton, Arthur L., Kerry de Hamsher y Abigail B. Sivan. 1983. Multilingual aphasia examination (2nd ed.). lowa City, IA: AJA Associates.

Bialystok, Ellen, Fergus I. Craik y Morris Freedman. 2007. «Bilingualism as a protection against the onset of symptoms of dementia». Neuropsychologia 45(2): 459-464.

Bialystok, Ellen, Fergus I. Craik y Gigi Luk. 2012. «Bilingualism: consequences for mind and brain». Trends in cognitive sciences 16(4): 240-250.

Bidelman, Gavin M., Jill E. Lowther, Sunghee H. Tak y Claude Alain. 2017. «Mild cognitive impairment is characterized by deficient brainstem and cortical representations of speech». Journal of Neuroscience 37(13): 3610-3620.

Duncan, Hillary D., Jim Nikelski, Randi Pilon, Jason Steffener, Howard Chertkow y Nathalie A. Phillips. 2018. "Structural brain differences between monolingual and multilingual patients with mild cognitive impairment and Alzheimer disease: Evidence for cognitive reserve». Neuropsychologia 109: 270-282

Folstein, Marshall F., Susan E. Folstein y Paul R. McHugh. 1975. "“Mini-mental state": a practical method for grading the cognitive state of patients for the clinician». Journal of psychiatric research 12(3): 189-198.

Freedman, Morris, Suvarna Alladi, Howard Chertkow, Ellen Bialystok, Fergus I. Craik, Natalie A. Phillips, Vasanta Duggirala, Bapi Raju Surampudi y Thomas H. Bak. 2014. "Delaying onset of dementia: are two languages enough?». Behavioural neurology 2014: 1-8. 
Guzmán-Velez, Edmarie y Daniel Tranel. 2015. «Does bilingualism contribute to cognitive reserve? Cognitive and neural perspectives». Neuropsychology, 29(1): 139-150.

Hughes, Charles P., Leonard Berg, Warren Danziger, Lawrence A. Coben y Ronald L. Martin. 1982. "A new clinical scale for the staging of dementia». The British journal of psychiatry 140(6): 566-572.

Illes, Judy. 1989. "Neurolinguistic features of spontaneous language production dissociate three forms of neurodegenerative disease: Alzheimer's, Huntington's, and Parkinson's». Brain and language 37(4): 628-642.

Jack Jr., Clifford R. y Ronald C. Petersen. 2000. «Structural imaging approaches to Alzheimer's disease». En Early Diagnosis of Alzheimer's Disease, ed. Leonard F. M. Scinto y Kirk R. Daffner (127-148). Totowa, NJ: Humana Press.

Karas, Giorgos, Philip Scheltens, Serge Rombouts, Ronald A. van Schijndel, Martin Klein, Bethany Jones, Wiesje van der Flier, Hugo Vrenken y Frederik Barkhof. 2007. «Precuneus atrophy in early-onset Alzheimer's disease: a morphometric structural MRI study». Neuroradiology 49(12): 967-976.

Lobo, Antonio, Pedro Saz y Guillermo Marcos. 2002. MMSE examen cognoscitivo mini-mental. Madrid: TEA Ediciones.

Martin, Alex y Paul Fedio. 1983. "Word production and comprehension in Alzheimer's disease: The breakdown of semantic knowledge». Brain and language 19(1): 124-141.

Murdoch, Bruce E., Helen J. Chenery, Vicki Wilks y Richard S. Boyle. 1987. «Language disorders in dementia of the Alzheimer type». Brain and language 31(1): 122-137.

Peña-Casanova, Jordi. 2005. Programa integrado de exploración neuropsicológica: Test Barcelona. Barcelona: Elsevier.

Perani, Daniela, Mohsen Farsad, Tommaso Ballarini, Francesca Lubian, Maura Malpetti, Alessandro Fracchetti, Giuseppe Magnani, Albert March y Jubin Abutalebi. 2017. "The impact of bilingualism on brain reserve and metabolic connectivity in Alzheimer's dementia». Proceedings of the National Academy of Sciences 114(7): 1690-1695.

Pfeffer, Robert I., Tom T. Kurosaki, C. H. Harrah Jr., Jeffrey M. Chance y S. Filos. 1982. «Measurement of functional activities in older adults in the community». Journal of gerontology 37(3): 323-329.

Ryu, Seon-Young, Min Jeong Kwon, Sang-Bong Lee, Dong Won Yang, Tae-Woo Kim, In-Uk Song, Po Song Yang, Hong Joo Kim y Ae Young Lee. 2010. «Measurement of precuneal and hippocampal volumes using magnetic resonance volumetry in Alzheimer's disease». Journal of Clinical Neurology 6(4): 196-203.

Schweizer, Tom A., Jenna Ware, Corinne E. Fischer, Fergus I. Craik y Ellen Bialystok. 2012. «Bilingualism as a contributor to cognitive reserve: Evidence from brain atrophy in Alzheimer's disease». Cortex 48(8): 991-996.

Serrano, Cecilia Mariela, Ricardo F. Allegri, Marina Drake, Judith Butman, Paula Harris, Carlos Nagle y Cristina Ranalli. 2001. «Versión abreviada en español del test de denominación de Boston: su utilidad en el diagnóstico diferencial de la enfermedad de Alzheimer». Revista de neurología 33(7): 624-627.

Steffener, Jason y Yaakov Stern. 2012. «Exploring the neural basis of cognitive reserve in aging». Biochimica et Biophysica Acta (BBA)-Molecular Basis of Disease 1822(3): 467-473.

Thomann, Philipp A., Christine Schläfer, Ulrich Seidl, Vasco Dos Santos, Marco Essig y Johannes Schröder. 2008. "The cerebellum in mild cognitive impairment and Alzheimer's disease-a structural MRI study». Journal of psychiatric research 42(14): 1198-1202. 
Vannini, Patrizia, Jane O'brien, Kraigher O'keefe, Maija Pihlajamäki, Peter Laviolette y Reisa A. Sperling. 2011. "What goes down must come up: role of the posteromedial cortices in encoding and retrieval». Cerebral Cortex 21(1): 22-34.

Wechsler, David. 1997. Wechsler memory scale (WMS-III). San Antonio, TX: Psychological corporation.

Wegiel, Jerzy, Henryk M. Wisniewski, Jerzy Dziewiatkowski, Eulalia Badmajew, Michal Tarnawski, Barry Reisberg, Bodgan Mlodzik, Jacqueline Mony y Douglas Craig Miller. 1999. «Cerebellar atrophy in Alzheimer's disease - Clinicopathological correlations». Brain research 818(1): 41-50.

Woumans, Evy, Patrick Santens, Anne Sieben, Jan Versijpt, Michaël Stevens y Wouter Duyck. 2015. «Bilingualism delays clinical manifestation of Alzheimer's disease». Bilingualism: Language and Cognition 18(03): 568-574. 